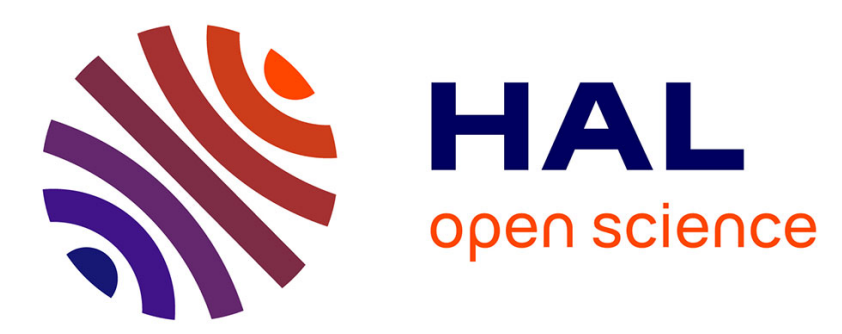

\title{
Experimental validation of Bessel beam generation using an inward Hankel aperture distribution
}

Mauro Ettorre, Santi Concetto Pavone, Massimiliano Casaletti, Matteo Albani

\section{To cite this version:}

Mauro Ettorre, Santi Concetto Pavone, Massimiliano Casaletti, Matteo Albani. Experimental validation of Bessel beam generation using an inward Hankel aperture distribution. IEEE Transactions on Antennas and Propagation, 2015, 63 (6), pp.2539-2544. 10.1109/TAP.2015.2419261 . hal-01154649

\section{HAL Id: hal-01154649 \\ https://hal.sorbonne-universite.fr/hal-01154649}

Submitted on 16 Oct 2015

HAL is a multi-disciplinary open access archive for the deposit and dissemination of scientific research documents, whether they are published or not. The documents may come from teaching and research institutions in France or abroad, or from public or private research centers.
L'archive ouverte pluridisciplinaire HAL, est destinée au dépôt et à la diffusion de documents scientifiques de niveau recherche, publiés ou non, émanant des établissements d'enseignement et de recherche français ou étrangers, des laboratoires publics ou privés. 


\title{
Experimental validation of Bessel beam generation using an inward Hankel aperture distribution
}

\author{
Mauro Ettorre, Member, IEEE, Santi C. Pavone, Student Member, IEEE, Massimiliano Casaletti, Member, IEEE, \\ and Matteo Albani, Senior Member, IEEE
}

\begin{abstract}
In this paper we prove experimentally that nondiffractive Bessel beams can be generated by using inward cylindrical traveling wave aperture distributions. An azimuthally invariant inward traveling wave distribution is defined over the aperture of a Radial Line Slot Array (RLSA) to launch a Bessel beam whose normal electric-field component assumes a truncated, zeroth-order Bessel function. An optimization procedure based on a holographic approach is used for tuning the position and size of the slots of the RLSA. The antenna is centrally fed by a coaxial probe transition. The final structure operates at $12.5 \mathrm{GHz}$. Full-wave simulations and measurements of the vertical component of the electric field show that a non-diffractive radiation is obtained within a range larger than 12 wavelengths in front of the antenna. The generated Bessel beam presents a stable half power beamwidth of about $20 \mathrm{~mm}$ within this range. The proposed system may open new opportunities for planar, low profile Bessel beam generators at millimeter waves, Terahertz, and optics.
\end{abstract}

Index Terms-Bessel beams, near field, focusing, radial line slot array, RLSA.

\section{INTRODUCTION}

D IFFRACTION is a basic physics phenomenon that limits the possibility to confine energy in a defined area in any radiating systems. Bessel beams are ideal solutions to Maxwell equations that do not suffer of diffractive spreading [1]. However, they require an infinite energy and radiating aperture. Nevertheless, practical implementations of Bessel beam generators have shown that the non-diffractive behaviour of such beams can be obtained within a defined limited area in front of the radiating aperture [2], known as non-diffractive range.

The solutions proposed so far to generate Bessel beams synthesize over a finite aperture a tangential field distribution shaped like a Bessel function either of the zero-th or first order. In particular, in the case of radial polarized zero-th order Bessel beams, the tangential field distribution is azimuthally invariant and is given by $J_{1}\left(k_{a} \rho\right)$, where $J_{1}($.$) is the first$ order Bessel function, $k_{a}$ is the transverse propagation constant

Manuscript received DATE; revised DATE.

The authors would like to thank the Ministère des Affaires Etrangéres et Européennes and Campus France (Galileo Projet) for their support and financial contribution.

M. Ettorre is with the Institut d'Electronique et de Télécommunications de Rennes (IETR), UMR CNRS 6164, Université de Rennes 1, 35042 Rennes Cedex, France. E-mail: mauro.ettorre@univ-rennes1.fr.

M. Casaletti is with the Sorbonne Universités, UPMC Univ Paris 06, UR2, L2E, F-75005, Paris, France (e-mail: massimiliano.casaletti@upmc.fr).

S. C. Pavone and M. Albani are with the Dipartimento di Ingegneria dell'Informazione Universitá degli Studi di Siena, Via Roma 56, 53100, Siena, Italy. E-mail: matteo.albani@ing.unisi.it associated to the Bessel beam, and $\rho$ is the radial coordinate of a cylindrical reference system [1].

Recently, several works [3]-[7] have highlighted the fact that Bessel beams are generated by the interference of two cylindrical traveling waves, or Hankel waves [4], one traveling outward and the other inward over a radiating finite aperture. Such combination is achieved in a small bandwidth due to the resonant nature of the obtained aperture distribution. However, in a recent paper, the authors have proven theoretically that Bessel beams can be generated by only inward cylindrical traveling waves [8] over a wide band of operation opening new opportunities for non-diffractive beams and localized pulses [9]. The goal of this paper is to confirm experimentally the conclusions drawn in [8].

In the following, we consider the generation of a propagating Bessel beam whose normal component takes on a zero-th order Bessel function $J_{0}\left(k_{a} \rho\right)$, with $k_{a}=0.4 k$ and $k$ the free space wavelength at the operating frequency of $f=12.5 \mathrm{GHz}$. This beam is generated by imposing an inward azimuthally invariant traveling wave distribution $H_{1}^{(1)}\left(k_{a} \rho\right)$ over a radiating aperture, where $H_{1}^{(1)}($.$) is the first order$ Hankel function of the first kind. Here the finite aperture is synthesized by a RLSA antenna. The optimization procedure outlined in [10] has been used for defining the size and position of the slots of the RLSA antenna. It is worth mentioning that a similar procedure has been adopted in [11] for the generation of a circular polarized Bessel beam by a RLSA structure [12]. However in this case the aperture distribution over the slotted plate of the RLSA consists of an azimuthally invariant zero-th order Bessel function.

The final antenna consists of a double grounded substrate where the slots of the RLSA are etched by laser ablation on one of the top metallic layer. The antenna is fed centrally by a coaxial feed. Measurement results of the vertical component of the electric field confirm the non-diffractive capability of the radiated field at the design frequency and the overall antenna design.

The paper is organized as follows. Section II is a brief summary of the results derived in [8]. Section III gives details on the design procedure used for synthesizing the required aperture field distribution with a RLSA structure. The prototype, full-wave and measurements results are shown in Section IV validating the antenna structure and the analytical derivations in [8]. Finally several conclusions are drawn in Section V. 


\section{BRIEF SUMMARY OF THE THEORETICAL RESULTS}

Let consider a finite circular radiating aperture of radius $a$ orthogonal to the $z$-axis of a cylindrical coordinate system $(\rho, \phi, z)$ with its origin at the center of the aperture. In the following, Transverse Magnetic (TM) modes with respect to the $z$-axis are considered; vectors are bold and a hat denotes a unit vector. An inward cylindrical wave is assumed for the tangential electric field on the radiating aperture: $\mathbf{E}_{\mathbf{t}}(\rho, \phi, z=$ $0)=E_{t}(\rho, z=0) \hat{\boldsymbol{\rho}}=H_{1}^{(1)}\left(k_{a} \rho\right) \hat{\boldsymbol{\rho}}$. The electric field radiated by the aperture is given by [13]

$$
\begin{gathered}
\mathbf{E}(\rho, z)=\frac{1}{4 \pi} \int_{\infty e^{-j \pi}}^{+\infty}\left[\frac{k_{\rho}}{k_{z}} H_{0}^{(2)}\left(k_{\rho} \rho\right) \hat{z}+j H_{1}^{(2)}\left(k_{\rho} \rho\right) \hat{\boldsymbol{\rho}}\right] \\
\cdot \widetilde{E}_{t}\left(k_{\rho}\right) e^{-j k_{z} z} k_{\rho} d k_{\rho} \\
\widetilde{E}_{t}\left(k_{\rho}\right)=-2 \pi j \int_{0}^{+\infty} E_{t}(\rho, z=0) J_{1}\left(k_{\rho} \rho\right) \rho d \rho
\end{gathered}
$$

where $k=2 \pi / \lambda$ is the free-space wavenumber, $k_{\rho}$ and $k_{z}=\sqrt{k^{2}-k_{\rho}^{2}}$ are the transverse and longitudinal spectral wavenumbers, respectively. $J_{n}($.$) and H_{n}^{(i)}($.$) are the n$-th order Bessel and Hankel functions of the $i$-th kind. Equation (2) is the Hankel-transform of the tangential field distribution over the aperture and, for the assumed inward finite cylindrical wave distribution, it is given by

$$
\begin{aligned}
\widetilde{E}_{t}\left(k_{\rho}\right)= & -\frac{4}{k_{a}\left(k_{\rho}^{2}-k_{a}^{2}\right)}\left\{\frac{k_{\rho}}{k_{a}}+\frac{\pi a}{2 j}\left[k_{\rho} H_{1}^{(1)}\left(k_{a} a\right) J_{0}\left(k_{\rho} a\right)\right.\right. \\
& \left.\left.-k_{a} H_{0}^{(1)}\left(k_{a} a\right) J_{1}\left(k_{\rho} a\right)\right]\right\}
\end{aligned}
$$

As an example related to the prototype realized in Sec. IV, let us consider a finite radiating aperture of radius $a=5.33 \lambda=$ $128 \mathrm{~mm}$ with an inward cylindrical field distribution with a transverse propagation constant $k_{a}=0.4 k=104.72 \mathrm{~m}^{-1}$ at the operating frequency of $f=12.5 \mathrm{GHz}$.

The first row of Fig. 1 shows the $z, \rho$ components and total electric field arranged in the $1^{\text {st }}, 2^{\text {nd }}$, and $3^{\text {rd }}$ column, respectively. As a comparison, the second row in Fig. 1 provides the field radiated by an ideal finite Bessel distribution over the same aperture and with the same transverse propagation constant of the inward Hankel wave: $\mathbf{E}_{\mathbf{t}}(\rho, \phi, z=$ $0)=J_{1}\left(k_{a} \rho\right) \hat{\boldsymbol{\rho}}$. The dashed lines in the figure represent the Geometrical Optics (GO) shadow boundaries arising from the aperture rim and due to the finiteness of the structure limiting the non-diffractive range to $z=a \cot \theta_{a} \simeq 12.21 \lambda$ $\left(\theta_{a}=\sin ^{-1}\left(k_{a} / k\right)=23.58^{\circ}\right)$. On the other hand, the dotted line in the inward Hankel case corresponds to the GO shadow boundary generated by the inward wave which, when passing through the caustic along the $z$-axis, becomes an outward conical wave [8].

Fig. 1 clearly shows that inward cylindrical aperture distribution creates a non-diffractive Bessel beam close to the antenna axis (namely in the triangular area delimited by the dotted and dashed lines, Fig. 1). On the other hand, the ideal Bessel distribution creates a non-diffractive radiation within a region in front of the aperture with a triangular cross section (area within the dashed line in the second row in Fig. 1). Nevertheless, the depth of focus of the two cases, i.e the nondiffractive range, is the same, and the shape of the realized
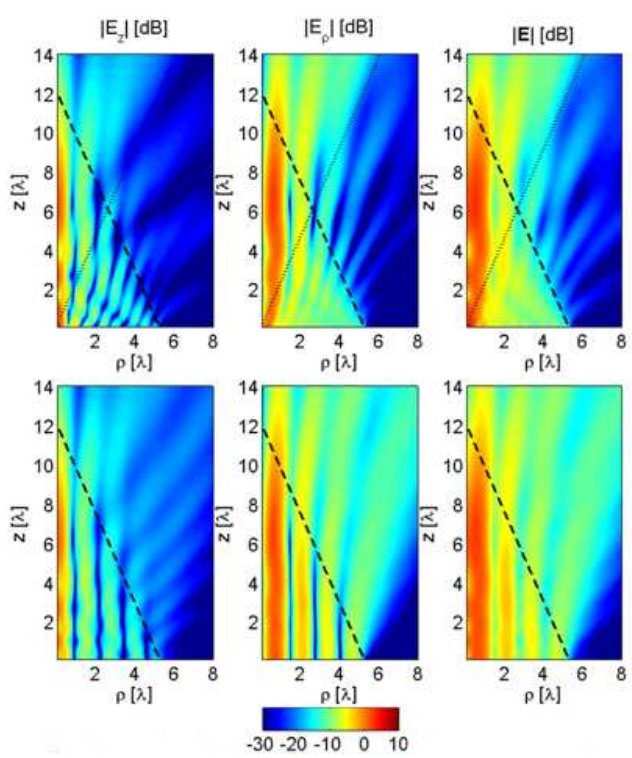

Fig. 1. Electric field radiated by a finite aperture with radius of $a=5.33 \lambda$. $\left|E_{z}\right|$ (1 ${ }^{\text {st }}$ column $),\left|E_{\rho}\right|\left(2^{\text {nd }}\right.$ column $)$ and total electric field amplitude $|\boldsymbol{E}|$ ( $3^{\text {rd }}$ column). The first and second row shows the field radiated by an inward Hankel and ideal Bessel distribution, respectively. The axes are normalized with respect to the wavelength $(\lambda)$ at the operating frequency $f$. The dashed line marks the GO boundaries in the two cases [8].

beam close to the $z$-axis is very similar. This demonstrates that inward Hankel aperture field distributions can launch efficiently a Bessel beam as standard Bessel distributions. It is worth mentioning that, for infinite radiating apertures, closed form expressions validate the numerical results presented in this section for the finite case, as presented and motivated in [8]. Here we have considered the finite case since closely related to a realistic implementation of a Bessel beam launcher.

\section{Synthesis OF the Aperture Field Distribution}

The required azimuthally invariant inward Hankel field aperture distribution is synthesized by a RLSA antenna at the operating frequency of $f=12.5 \mathrm{GHz}$ using the optimization procedure outlined in [10]. This procedure controls the position and size of each slot of the RLSA antenna in such a way to create, over the radiating aperture, the equivalent magnetic current distribution associated to the inward Hankel wave: $\mathbf{M}_{\mathbf{0}}(\rho)=M_{0}(\rho) \widehat{\phi}=-E_{\rho}(\rho) \widehat{\phi}=H_{1}^{(1)}\left(k_{a} \rho\right) \widehat{\phi}$. In addition, the antenna spillover efficiency (ratio between the radiated and accepted power by the antenna) is maximized during the design to prevent spurious radiation from the edge of the structure. In the following, an efficiency larger than $95 \%$ has been imposed as constraint [10]. The slots of the RLSA and the associated magnetic dipole moments are oriented along $\phi$ as the equivalent magnetic current distribution. A fast and accurate in-house Method of Moments (MoM) [14][17] is used during the optimization procedure to evaluate the magnetic dipole moment of each slot and overall radiation performance.

The final structure is shown in Fig. 2. The position and size of the slots are given in Table I. The width of the slots $(w)$ is equal to $1.2 \mathrm{~mm}$. A spillover efficiency larger than $97 \%$ 
TABLE I

POSITION AND DIMENSIONS (in $\mathrm{mm}$ ) OF THE SLOTS IN EACH RING OF THE RLSA

\begin{tabular}{|c|c|c|c|c|c|c|c|c|c|c|c|}
\hline Ring number & 1 & 2 & 3 & 4 & 5 & 6 & 7 & 8 & 9 & 10 & 11 \\
\hline$l_{i}$ & 7.42 & 7.22 & 7.38 & 7.59 & 7.19 & 7.90 & 7.55 & 8.12 & 7.72 & 9.05 & 10.26 \\
\hline$\rho_{i}$ & 8.96 & 23.20 & 34.24 & 47.38 & 58.62 & 71.54 & 82.46 & 95.18 & 106.50 & 118.03 & 127.02 \\
\hline n. of slots & 5 & 13 & 20 & 28 & 34 & 42 & 48 & 55 & 62 & 69 & 74 \\
\hline
\end{tabular}

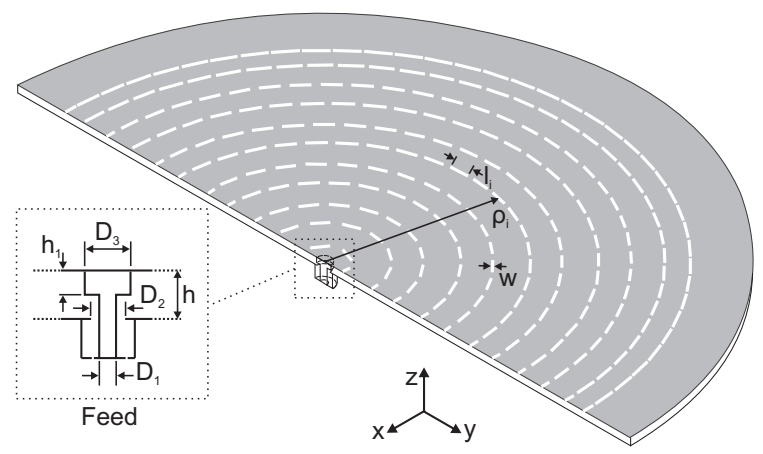

Fig. 2. Cross section of the RLSA antenna and geometrical details of the coaxial probe transition. The slots on each ring of the RLSA have the same length $\left(l_{i}\right)$ and are uniformly distributed along $\phi$. The width of the slots $(w)$ is constant.

and an average aperture distribution error lower than $4.5 \%$ are achieved at the end of the optimization loop [10]. The antenna is fed by a coaxial probe transition as shown in the inset in Fig. 2 with geometrical dimensions equal to: $D_{3}=6.96 \mathrm{~mm}$, $D_{2}=4.52 \mathrm{~mm}, D_{1}=1.27 \mathrm{~mm}, h=3.175 \mathrm{~mm}$, and $h_{1}=$ $1.88 \mathrm{~mm}$.

To estimate the quality of the obtained aperture field distribution, Fig. 3 shows the normalized $z$-component of the electric field at $z=a / 2 \cot \theta_{a} \simeq 6.11 \lambda=146.64 \mathrm{~mm}$ radiated by the RLSA antenna along the $x$ - and $y$-axes and by the ideal inward Hankel wave. In the ideal case, the field is azimuthally invariant. The main and lateral lobes of the field radiated by the RLSA antenna are in very good agreement with the ideal case demonstrating the good accuracy of the synthesized aperture distribution and overall RLSA antenna design. The discrepancies for field levels lower than $-30 \mathrm{~dB}$ are considered residual effects of the achieved average aperture distribution error. It is worth noting that the plane at $z=146.64 \mathrm{~mm}$ passes through the intersection of the two GO boundaries shown in Fig. 1, and corresponds to the maximum extension of the nondiffractive zone along the radial direction for inward Hankel distributions, as outlined in Section II.

\section{PRototype And Experimental Results}

The structure optimized in Section III has been manufactured and measured at IETR. The final antenna is shown in Fig. 4. The antenna is made on a double grounded Neltec NY9217 substrate with permittivity $\epsilon_{r}=2.17$ and thickness $h=3.175 \mathrm{~mm}$ (refer to Fig. 2). Laser ablation has been used to etch the slots of the RLSA on the upper face of the substrate. The prototype is fed on the back by a SMA connector using the coaxial probe transition shown in Fig. 2. Fig. 5 shows the simulated and measured input reflection coefficient. The antenna is matched at the operating frequency

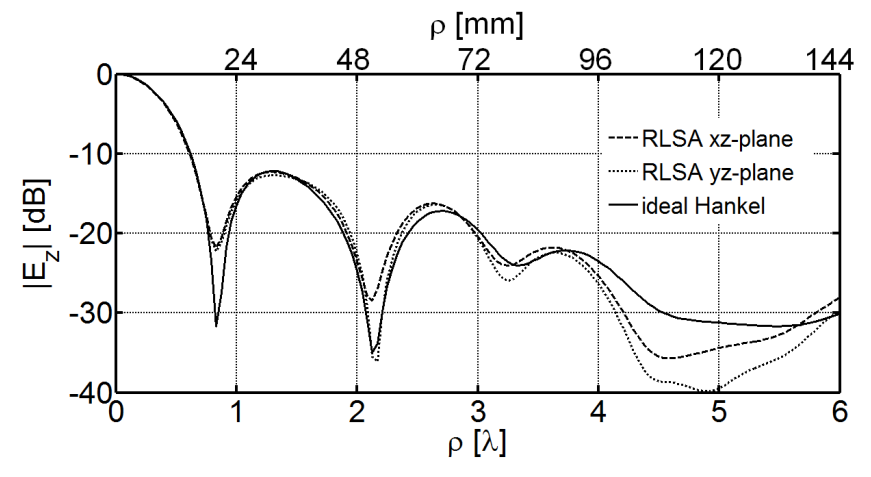

Fig. 3. Normalized $E_{z}$ component of the electric field at $z=a / 2 \cot \theta_{a}$ radiated by the synthesized RLSA antenna along the $x-$ and $y-$ axes. The field radiated by an ideal inward Hankel wave is provided for comparison.

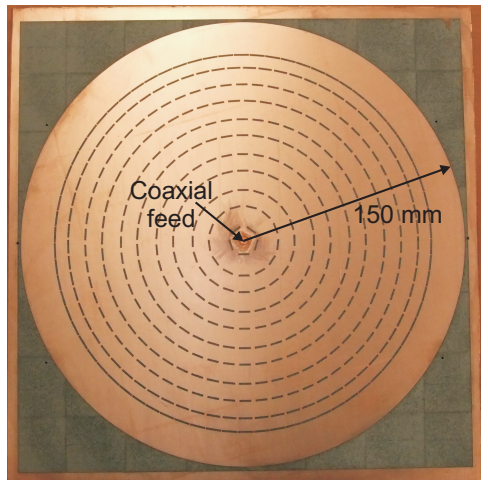

Fig. 4. Top view of the RLSA antenna. The slots have been etched by laser ablation. The coaxial probe transition is visible at the center of the prototype. Note that a residual rim of copper is present at the edges of the structure used for fixing purposes without any effects on the antenna performance.

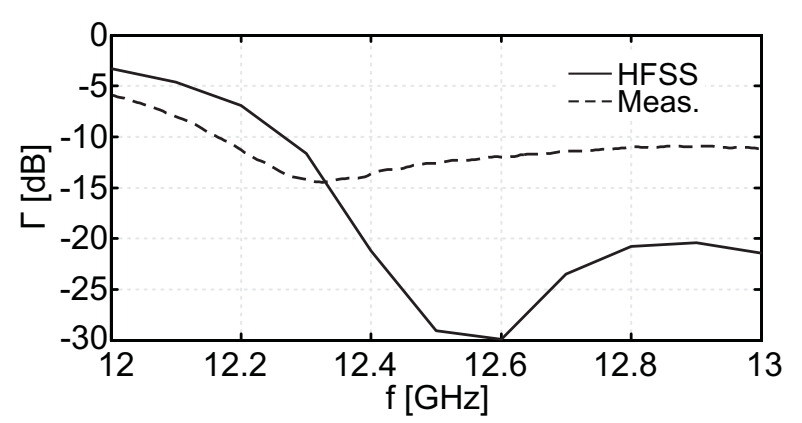

Fig. 5. Measured and simulated input reflection coefficient of the RLSA antenna. 


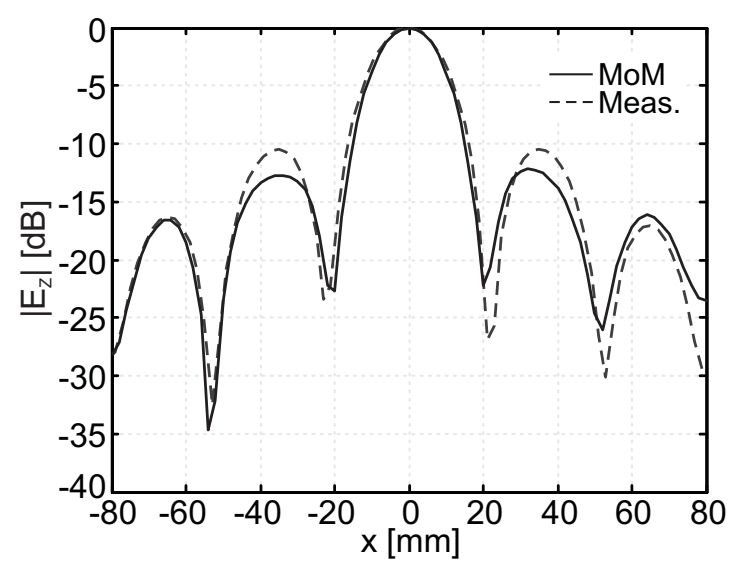

(a)

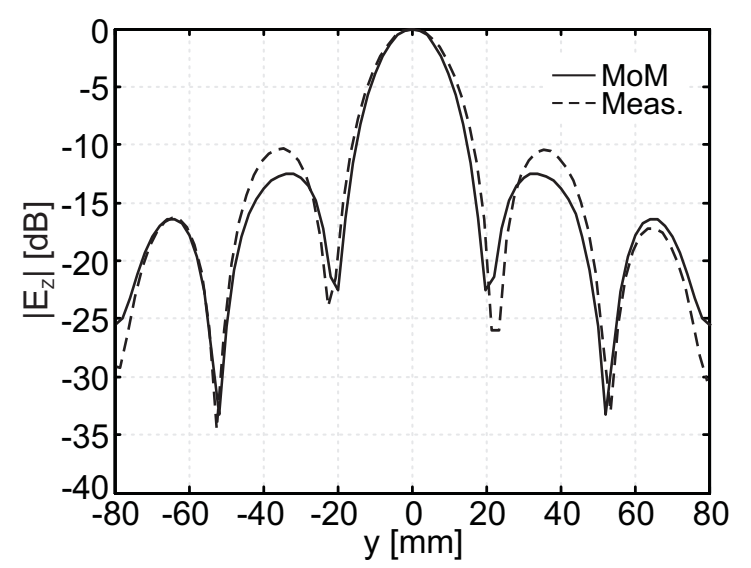

(b)

Fig. 6. Normalized $E_{z}$ component at the operating frequency $f=12.5 \mathrm{GHz}$ at $z=150 \mathrm{~mm}$. (a) $x$-axis. (b) $y$-axis. Measurements and MoM results are compared along the two axes.

and within the band 12.16-13.00 GHz. However, the measured input reflection coefficient is shifted downward $(\approx 1.8 \%)$ and presents a higher level with respect to the numerical results [18], but still below the required $-10 \mathrm{~dB}$ level. These differences can be attributed to fabrication constraints related to the proposed probe transition. In fact, a milling process is used to create within the substrate the disk of diameter $D_{3}$ in Fig. 2, which is afterwards metalized and connected to the inner conductor of the SMA connector. An accuracy of about $100 \mu \mathrm{m}$ is expected from the milling process.

The normal component of the electric field $E_{z}$ radiated by the antenna has been measured using a short electric probe attached to an automatically controlled 2D translation stage. The short electric probe is made out of a semi-rigid coaxial cable (UT-85) by removing the external conductor and dielectric insulator. The inner conductor extends for a length of $\lambda / 10=2.5 \mathrm{~mm}$ beyond the outer conducting shield.

The normalized $E_{z}$ component at the operating frequency $f=12.5 \mathrm{GHz}$ at $z=150 \mathrm{~mm}$ along the $x$ - and $y$-axis

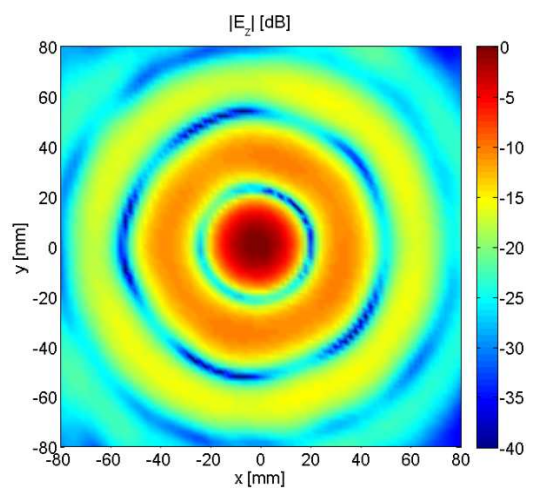

(a)

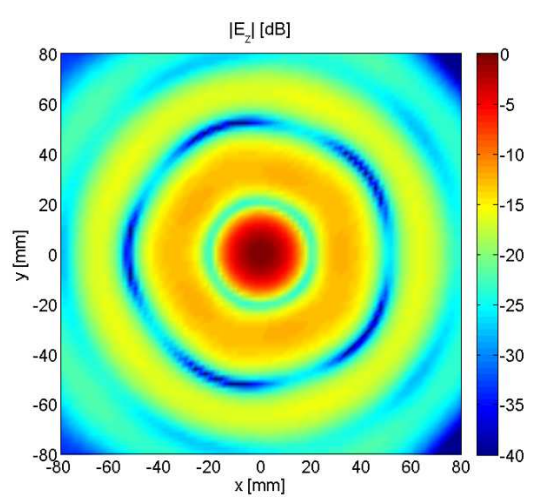

(b)

Fig. 7. Normalized $E_{z}$ component at the operating frequency $f=12.5 \mathrm{GHz}$ at $z=150 \mathrm{~mm}$. (a) Measurements. (b) MoM results.

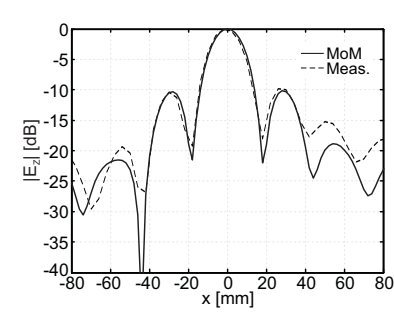

(a)

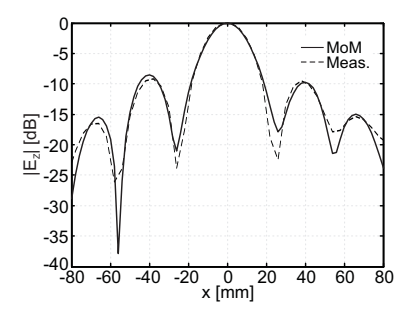

(c)

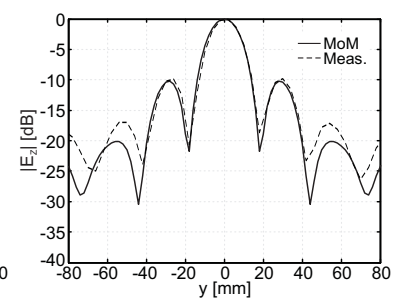

(b)

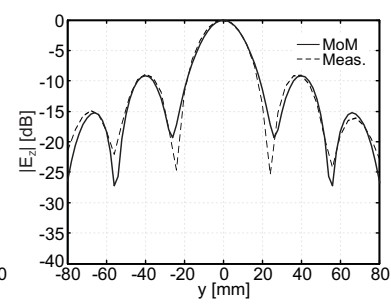

(d)
Fig. 8. Normalized $E_{z}$ component at the edges of the considered frequency band at $z=150 \mathrm{~mm}$. (a) $x$-axis at $12.2 \mathrm{GHz}$. (b) $y$-axis at $12.2 \mathrm{GHz}$. (c) $x$-axis at $12.7 \mathrm{GHz}$. (d) $y$-axis at $12.7 \mathrm{GHz}$. 
(a)

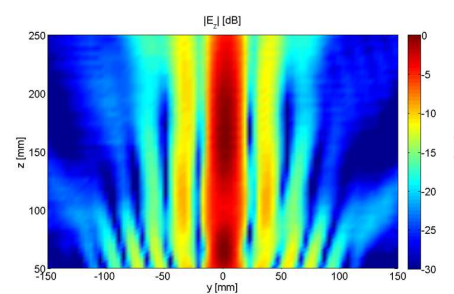

(c)

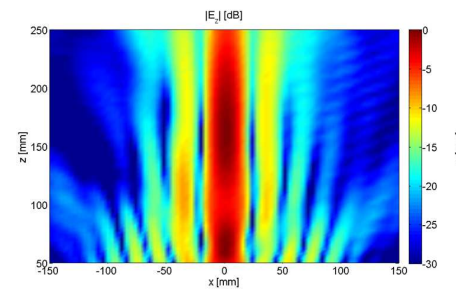

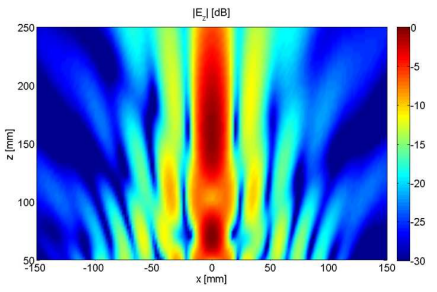

(b)

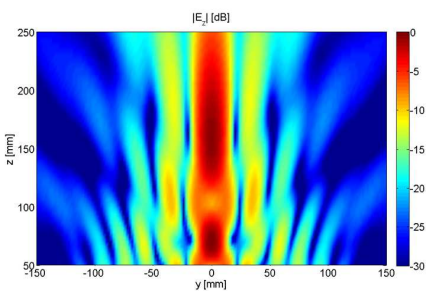

(d)
Fig. 9. Normalized $E_{z}$ component at the operating frequency $f=12.5 \mathrm{GHz}$ along the planes normal to the RLSA antenna. (a) Measurements along the $x z$-plane. (b) MoM results along the $x z$-plane. (c) Measurements along the $y z$-plane. (d) MoM results along the $y z$-plane.

is shown in Figs. 6a and b, respectively. These field cuts have been obtained by measurement data over an area of $160 \times 160 \mathrm{~mm}^{2}$ with a space step of $2 \mathrm{~mm}$. Good agreement is achieved between the measured and simulated results. However, a small difference is observed in the side lobe level (SLL) of the measured fields with respect to the MoM results in both field cuts. Such differences may be due to the fabrication tolerances related to the etching and milling processes used for antenna on the order of $50 \mu \mathrm{m}$ and $100 \mu \mathrm{m}$, respectively. Fig. 7 reports the measured and MoM 2D field plots for the normalized $E_{z}$ components at $z=150 \mathrm{~mm}$. Also in this case, a good agreement can be observed between the measured and simulated results validating the overall antenna design.

The radiated field has been measured in the band 12.212.7 $\mathrm{GHz}$ corresponding to an input reflection coefficient lower than $-10 \mathrm{~dB}$ (refer to Fig. 5). Fig. 8 shows the measured and simulated normalized $E_{z}$ component at $z=150 \mathrm{~mm}$ at the edges of the considered band. It can be noticed that the field pattern is preserved within the considered band and there is a good agreement between simulation and measurements.

The non-diffractive behavior of the generated beam has been verified by measuring the field along the $x z$ - and $y z$-planes over an area of $300 \times 200 \mathrm{~mm}^{2}$ with a step of $4 \mathrm{~mm}$. Fig. 9 shows the measured and simulated 2D plots of the normalized $E_{z}$ field along the $x z$ - and $y z$-planes. The main beam of the generated radiation does not change within the considered range $(z=50-250 \mathrm{~mm})$. This is further highlighted by the contour plots in Fig. 10 for the measured and simulated main beam along the two considered planes. The $3 \mathrm{~dB}$ beamwidth of the generated Bessel beam is about $20 \mathrm{~mm}$ and roughly constant within the measured area in both planes.

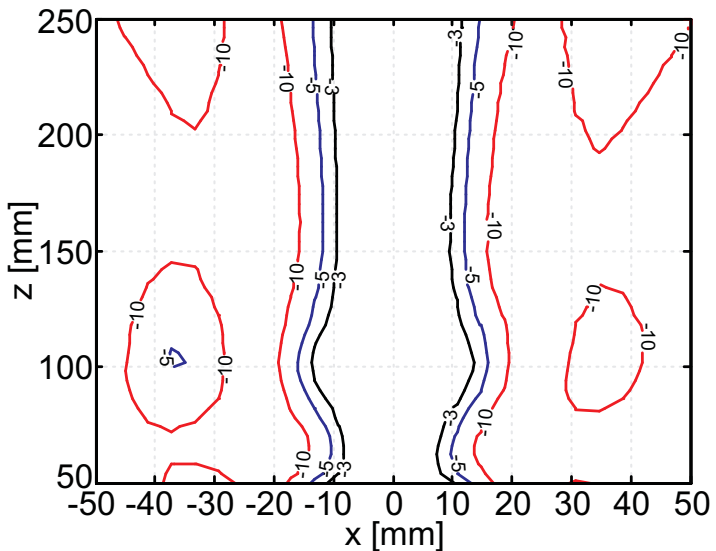

(a)

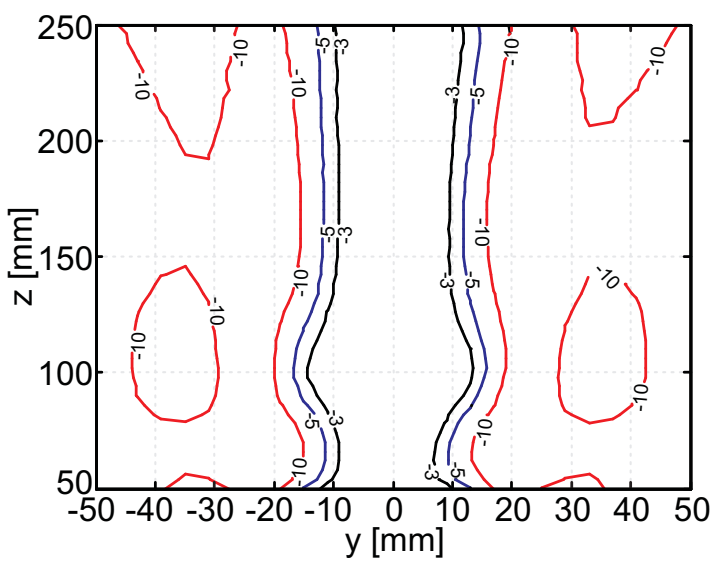

(b)

Fig. 10. Contour plots for the measured normalized $E_{z}$ component at the operating frequency $f=12.5 \mathrm{GHz}$. (a) $x z$-plane. (b) $y z$-plane.

The operation of the structure within the considered band $(12.2-12.7 \mathrm{GHz})$ is then analyzed. The measured 2D plots of the normalized $E_{z}$ field along the $x z$ - and $y z$-planes at 12.2 $\mathrm{GHz}$ and $12.7 \mathrm{GHz}$ are provided in Fig. 11. It is possible to observe that the main beam of the radiated field does not change within the considered space range and operating frequency band validating the large band operation of the proposed structure [8]. For brevity, only measurement results are shown but also for these cases there is a good agreement with simulation results.

\section{CONCLUSION}

We have presented and validated experimentally that nondiffractive Bessel beams can be generated by using inward cylindrical aperture field distributions. The inward Hankel distribution is synthesized over the radiating aperture by using a recent developed optimization procedure for RLSA antennas. The final RLSA structure is planar and does not require any resonant aperture distribution to generate the non-diffractive 


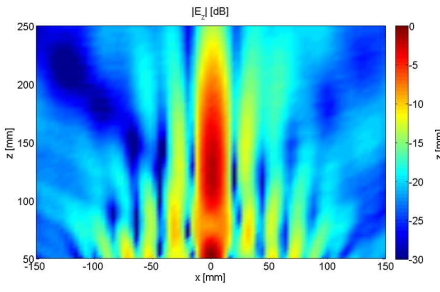

(a)

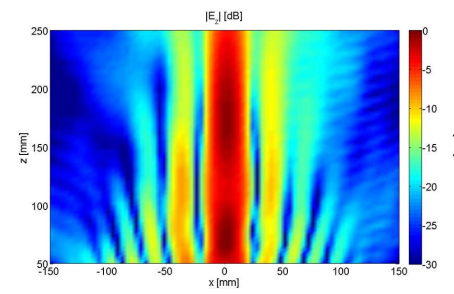

(c)

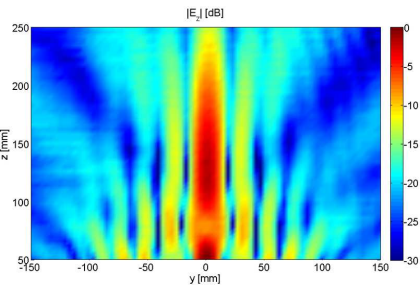

(b)

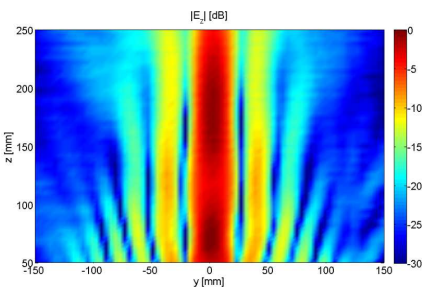

(d)
Fig. 11. Normalized measured $E_{z}$ component at the edges of the considered frequency band. (a) $x z$-plane at $12.2 \mathrm{GHz}$. (b) $y z$-plane at $12.2 \mathrm{GHz}$. (c) $x z$ plane at $12.7 \mathrm{GHz}$. (d) $y z$-plane at $12.7 \mathrm{GHz}$.

radiation as in previous works. The radiated field well approximate a Bessel beam close to the antenna axis within a non-diffractive range which is the same of a standard Bessel aperture distribution of the same size. A prototype based on a standard and low cost printed circuit board process has been manufactured and tested. The synthesized inward aperture distribution has a transverse propagation constant equal to $0.4 k$ at $12.5 \mathrm{GHz}$. Measurement results for the normal component of the electric field validate the design and prove that the main beam of the radiated field exhibits an almost constant width within the measured area $(z=50-250 \mathrm{~mm})$. In this range, the $3 \mathrm{~dB}$ beamwidth of the generated Bessel beam is roughly $20 \mathrm{~mm}$. The proposed structure may open new possibilities for the generation of non-diffractive radiation and localized pulses at millimeter waves, Terahertz, and optics.

\section{REFERENCES}

[1] J. Durnin, "Exact Solutions for Nondiffracting Beams. I. The Scalar Theory," J. Opt. Soc. Am. A., vol. 4, no. 4, pp. 651-654, Apr. 1987.

[2] M. Lapointe, "Review of Non-Diffracting Bessel Beam Experiments," Opt. Las. Tech., vol. 24, no. 6, pp. 315-321, Dec. 1992.

[3] S. Chávez-Cerda, "A new approach to Bessel beams," J. Mod. Opt., vol. 46, no. 6, pp. 923-930, 1999.

[4] S. Chávez-Cerda, and G. H. C. New, "Evaluation of focused Hankel waves and Bessel beams," Opt. Commun., vol. 181, no. 4-6, pp. 369377, Jul. 2000.

[5] M. Ettorre and A. Grbic, "Generation of propagating Bessel beams using leaky-wave modes," IEEE Trans. Antennas Propag., vol. 60, no. 8, pp. 3605-3605, Aug. 2012.

[6] M. Ettorre, S. M. Rudolph and A. Grbic, "Generation of propagating Bessel beams using leaky-wave modes: experimental validation," IEEE Trans. Antennas Propag., vol. 60, no. 6, pp. 2645-2653, Jun. 2012.

[7] M. F. Inami and A. Grbic, "Generating Bessel beams using an electrically-large annular slot," in Proceedings of IEEE AP-S/URSIUSNC Symposium, (IEEE 2013).
[8] M. Albani, S. C. Pavone, M. Casaletti, and M. Ettorre, "Generation of non-diffractive Bessel beams by inward cylindrical traveling wave aperture distributions," Opt. Express, vol. 22, no. 15, pp. 18354-18364, Jul. 2014.

[9] H. E. Hernandez-Figueroa, E.Recami, and M. Zamboni-Rached, Nondiffractive waves, J.Wiley-VCH, Berlin, 2013.

[10] M. Ettorre, M. Casaletti, G. Valerio, R. Sauleau, L. Le Coq, S. C. Pavone, and M. Albani, "On the near-field shaping and focusing capability of a radial line slot array," IEEE Trans. Antennas Propag., vol. 62, no. 4, pp. 1991-1999, Apr. 2014

[11] M. Albani, A. Mazzinghi, and A. Freni, "Automatic design of CP-RLSA antennas," IEEE Trans. Antennas Propag., vol. 60, no. 12, pp. 55385547, Dec. 2012.

[12] A. Mazzinghi, M. Balma, D. Devona, G. Guarnieri, G. Mauriello, M. Albani, and A. Freni, "Large depth of field pseudo-Bessel beam generation with a RLSA antenna," IEEE Trans. Antennas Propag., vol. 62, no. 8, pp. 3911-3918, Aug. 2014.

[13] L. B. Felsen and N. Marcuvitz, Radiation and Scattering of Waves, IEEE Press Series on Electromagnetic Wave Theory, Piscataway, NJ, 1994.

[14] M. Casaletti, R. Sauleau, M. Ettorre, and S. Maci, "Efficient analysis of metallic and dielectric posts in parallel plate waveguide structures," IEEE Trans. Microw. Theory Tech., vol. 60, no. 10, pp. 2979-2989, Oct. 2012.

[15] M. Casaletti, G. Valerio, J. Seljan, M. Ettorre, and R. Sauleau, "A full-wave hybrid method for the analysis of multilayered SIW-based antennas," IEEE Trans. Antennas Propag., vol. 61, no. 11, pp. 55755588, Nov. 2013.

[16] M. Albani, G. Lo Cono, R. Gardelli, and A. Freni, "An efficient fullwave method of moments analysis for RLSA antennas," IEEE Trans. Antennas Propag., vol. 54, no. 8, pp. 2326-2336, Aug. 2006.

[17] M. Albani, A. Mazzinghi, and A. Freni, "Rigorous MoM analysis of finite conductivity effects in RLSA antennas," IEEE Trans. Antennas Propag., vol. 59, no. 11, pp. 4023-4032, Nov. 2011.

[18] Ansoft HFSS version 15.0, 1984-2014 Ansoft Corporation.

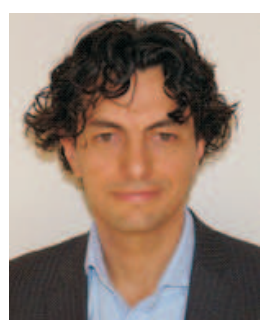

Mauro Ettorre (M'08) was born in Tricarico, Matera, Italy, in 1979. He received the laurea degree (summa cum laude) in telecommunication engineering and the Ph.D. degree in electromagnetics from the University of Siena, Italy, in 2004 and 2008 respectively.

During his Master's degree studies he spent five months at the Technical University of Denmark (DTU), Lyngby, Denmark. Part of his PhD has been developed at the Defence, Security and Safety Institute of the Netherlands Organization for Applied Scientific Research (TNO), The Hague, The Netherlands, where afterwards he worked as antenna researcher. From 2008 to 2010, he was a Postdoctoral Fellow at the Institut d'Electronique et de Télécommunications de Rennes (IETR), Université de Rennes 1, France. From 2010 to 2011, he was a visiting scholar at the Radiation Laboratory, Department of Electrical Engineering and Computer Science, University of Michigan, Ann Arbor, USA. Since October 2010, he has been appointed permanent CNRS researcher at IETR.

His research interests include the analysis and design of leaky-wave antennas, periodic structures, compact planar antennas and wireless power transmission systems.

Dr. Ettorre received the Young Antenna Engineer Prize during the 30th ESA Antenna Workshop 2008 in Noordwijk, The Netherlands. 


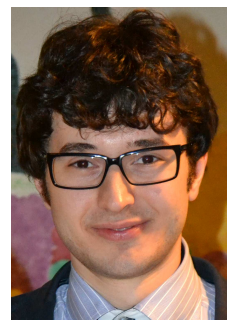

Santi Concetto Pavone was born in Patti (ME), Italy, in 1988. He received the Bachelor's Degree (B.Sc.) in Electronics Engineering (Cum Laude) in 2010 and the Master's Degree (M.Sc.) in Electronics Engineering (Cum Laude) in 2012, both from the University of Messina, Italy. During his Master's Thesis, he designed a Low Noise Amplifier and an Integrated Antenna for Millimeter Waves Applications (60 GHz), using a CMOS SOI $65 \mathrm{~nm}$ Technology for Analog ICs. Currently, he is a Ph.D. Student in Information Engineering and Science (IES), research line in Electromagnetics Engineering, at the University of Siena, Italy. His research interests include leaky-wave antennas, focusing systems at microwaves, mw electronics and optoelectronics. In 2014, S. C. Pavone won the IEEE AP-S Student Award Chapter Central-Southern Italy.

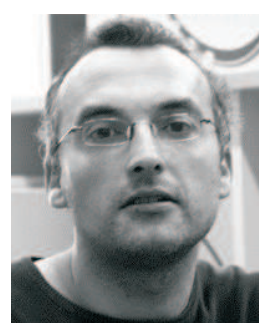

Massimiliano Casaletti was born in Siena, Italy, in 1975. He received the Laurea degree in telecommunications engineering and the Ph.D. degree in information engineering from the University of Siena, Italy, in 2003 and 2007, respectively. From September 2003 to October 2005, he was with the research center MOTHESIM, Les Plessis Robinson (Paris, FR), under EU grant RTN-AMPER (RTN: Research Training Network, AMPER: Application of Multiparameter Polarimetry). He has been Research Associate at the University of Siena, Italy from November 2006 until October 2010 and a Postdoctoral Researcher from November 2010 to August 2013 at the IETR (Institut d'Electronique et des Télécommunications de Rennes) at University of Rennes 1, Rennes, France. $\mathrm{He}$ is currently an Associate Professor at University Pierre-et-Marie-Curie (UPMC), Paris, France.

His research interests include numerical methods for electromagnetic (scattering, antennas and microwave circuits), metasurface structures, field beam expansion methods, electromagnetic band-gap structures.

Dr. Casaletti was a co-recipient of the Best Poster Paper Award at the 3rd European Conference on Antennas and Propagation (EuCAP-2009), Berlin, Germany, and the recipient of an Honorable Mention for Antenna Theory at EuCAP-2010, Barcelona, Spain, and the Best Paper Award on Antenna Theory at EuCAP-2011, Rome, Italy.

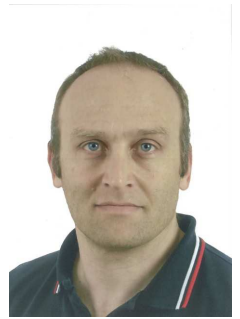

Matteo Albani (M98SM10) received the Laurea degree in electronic engineering and Ph.D. degree in telecommunications engineering from the University of Florence, Florence, Italy, in 1994 and 1999, respectively.

$\mathrm{He}$ is currently an Adjunct Professor with the Information Engineering and Mathematics Department, University of Siena, Siena, Italy, where he is also Director of the Applied Electromagnetics Laboratory. From 2001 to 2005, he was an Assistant Professor with the University of Messina, Messina, Italy. He coauthored more than 60 journal papers and more than 160 conference papers. His research interests are in the areas of high-frequency methods for electromagnetic scattering and propagation, numerical methods for array antennas, antenna analysis and design, metamaterials.

Dr. Albani received the G. Barzilai Young Researcher Best Paper Award, at the XIV RiNEM, Ancona, Italy 2002, and the URSI Commission B Young Scientist Award at 2004 URSI EMTS, Pisa, Italy. He was Co-author and Advisor of the winners of the Best Paper Award, at the First European AMTA Symp. 2006, Munich, Germany and of the "3rd Prize Young Scientist Best Paper Award" 2010 URSI EMTS, Berlin, Germany. With his co-authors. he was awarded with the Antenna Theory Best Paper at the EuCAP 2014 in den Haag, the Netherlands.

$\mathrm{He}$ is a member of EURAAP and URSI. 